\title{
LA LEY ORGANICA DE REGIMEN MUNICIPAL DE VENEZUELA (20 de septiembre de 1988)
}

\author{
POR \\ José Manuel Castells Arteche
}

\begin{abstract}
sumario: I. Breve historia.-II. Claves superestructurales.-III. Principales innoVACIONES de LA LEY ORgánICA de RÉGIMEN MUNICIPAL de 1988: 1. Las fuentes del ordenamiento local. 2. Estructuras locales. 3. El modelo organizativo y de funcionamiento. 4. El plano de las competencias. 5. La hacienda pública municipal. 6. La participación política en el régimen municipal.-IV. EL DIFíCIL DECURSO DE LA LEY.
\end{abstract}

\section{BREVE HISTORIA}

En un país de contrastes como es la actual Venezuela, una de las asignaturas pendientes más vivamente denotables, es la referente a un real desarrollo de la vida local. No es que falten datos normativos para reflejar una activa savia municipal; ya la Constitución de 1857, había citado, ya se verá con qué finalidad, al poder municipal entre los poderes públicos (1). Por encima de declaraciones, con todo, es perceptible la habitual contradicción entre el énfasis de la letra impresa y la triste realidad, que desconoce paladinamente retóricas manifestaciones.

La actual Constitución de 23 de enero de 1961, que ha supuesto la plasmación de la normalidad de un sistema democrático una vez periclitada la dictadura de Pérez Jiménez, consagraba varios artículos a la organización de las corporaciones locales, estableciendo innovadoramente (2) que los municipios constituían «la unidad política de base y de autonomía en la organización nacional»; autonomía que el artículo $\mathbf{2 9}$ definía por el dato de su afectación a: «1. ${ }^{\circ}$ la elección de sus autoridades; $2 .^{\circ}$ la libre gestión de las materias de su competencia, y $3 .^{\circ}$ la creación, la recaudación y la inversión de sus ingresosil.

La doctrina ha destacado el retraso posconstitucional en la regulación de los municipios democráticos, puesto que hasta la Ley Orgánica de 18 de agosto de 1978, no se entró en la ordenación de las entidades locales, que siguieron rigiéndose por la normativa anterior a la Constitución de 1961, claramente inserta en el más puro desconocimiento de la vida local; comenzando por la figura

(1) Al respecto, A. R. BREWER-CARIAS: Las Constituciones de Venezuela. Madrid, 1985. p. 363

(2) Véase F. MODERNE: «El problema de la reforma municipal en Venezuela», en el número 1 de la Revista Vasca de Administración Pública, p. 21.

REVISTA DE ESTUDIOS. -9 
misma del municipio, reemplazado por sucedáneos de dudoso valor.

Tampoco esta ley, votada durante el anterior mandato presidencial de Carlos Andrés Pérez, sirvió para actualizar y concretar debidamente los parámetros propios de un municipio democrático (3), limitándose a regular las municipalidades a nivel de distrito provenientes del régimen predemocrático, prorrogando su transitoriedad hasta 1984; sí que se posibilitó, bien que de forma excesivamente tardía, las elecciones a nivel de distrito (1979). Del mismo modo que tampoco se llevaron a cabo los reglamentos ejecutivos pertinentes, salvo en algún plano sectorial, como el que atañía a las asociaciones de vecinos.

En este contexto se promulga la reciente Ley Orgánica de Régimen Municipal (20 de septiembre de 1988), cuyo artículo 186 establece su entrada en vigencia a partir del 15 de junio de 1989 , salvo en los preceptos relativos a la elección de los representantes de "los poderes públicos municipales», que en concordancia con una precedente Ley Orgánica del sufragio (29 de marzo de 1984). debía aplicarse a partir de la publicación de la Ley Orgánica.

Ley Orgánica (4) que por primera vez ha desarrollado el municipio democrático tal como principalmente lo perfiló la Constitución; significando, del mismo modo, una adaptación a modelos comparados ya arraigados y una racionalización de unas estructuras ancladas en un pretérito no demasiado brillante. El futuro clarificará el alcance exacto de la reforma; nos toca avizorar ahora, unas claves explicativas de la medida, así como aludir a unas puntuales innovaciones.

\section{CLAVES SUPERESTRUCTURALES}

Venezuela ha sido el triste escenario de una permanente situación de guerra civil, entreveradas de «caudillajes» militares (5), que prácticamente abarca de forma constante, desde el fin de la colonia hasta el advenimiento de la democracia, en los años finales de la década de los cincuenta (6). No presenta margen a la duda, que esta secular inestabilidad no se prestaba a proporcionar

(3) En opinión de BREWER-CARIAS: Introducción General a la Ley orgánica de Régimen Municipal. Caracas, 1988, p. 11.

(4) Las leyes orgánicas, según el artículo 163 de la Constitución, poseen esta calificación bien por virtud de la propia Constitución, sea por reclamarlo las dos cámaras, mediante acuerdo adoptado por mayoría absoluta en el inicio del proceso.

(5) Una excelente descripción de la penúltima de estas dictaduras, la del general Gómez en la obra USLAR PIETRI: Oficio de Difuntos (Caracas, 1976).

(6) Para una visión global de este azaroso tiempo, incluso desde el prisma latinoamericano, la obra de SALCEDO-BASTARDO: Historia Fundamental de Venezuela. Caracas, 1982. 
facilidades para un adecuado desenvolvimiento del sistema local, último peldaño del botín de la autocracia gobernante. Nada nuevo bajo el sol, al menos por estas latitudes hispanas, con las secuelas habituales de corrupción, caciquismo e inoperancia en la gestión.

Sin embargo, la temprana Constitución de 1811 habíase decantado por una Confederación para Venezuela, siguiendo el modelo norteamericano, que con el tiempo llegaría a conformar a la República de Venezuela en un Estado federal. Bien es cierto que, como ha señalado BREWER-CARIAS puede denominarse este sistema con mayor acierto, como "una federación centralizada» (7), en el que el centralismo larvado representa el auténtico y real poder político, al margen nominalismos estéticos.

Curiosamente «el poder municipal» significará a medidados del pasado siglo, un instrumento de la reacción centralista contra los poderes de los Estados federados, buscando «la eliminación de las provincias» por medio del escalón de base. Una vez más se asiste al interesado juego del poder central, que con el objetivo de afianzar sus intereses no duda en lanzar la alternativa municipal.

En suma, la presente Constitución reconoce la existencia de tres niveles de poderes públicos: el nacional, el estatal y el municipal; o lo que es lo mismo, el poder nacional que corresponde a la República; los 20 Estados, más un distrito federal y dos territorios federales; un amplio elenco de entidades agrupadas en el poder municipal, que se benefician de las relaciones contradictorias entre los dos primeros poderes.

En el interior de estos parámetros, no puede menos de mencionarse, por afectar de forma bien directa a la normativa en cuestión, de forma singular en el ámbito de la hacienda municipal, a la aguda crisis económica que asola a Venezuela, país por otra parte que posee enormes materias primas. Crisis que ha ocasionado estallidos de una rara violencia, como los desarrollados en determinados barrios caraqueños en febrero de este mismo año, pero que en sus ineluctables secuelas pueden convertir en papel mojado cualquier pretensión reformista, como la que se esboza en el analizado espacio.

Finalmente, el turno de partidos entre AD (Acción Democrática) y COPEI (Comité de Organización Política Electoral Independiente), que se alternan en el poder después de la caída del último dictador, lejos de suponer una estabilidad democrática, han enviciado el área local, sin que corrientes alternativas de progreso hayan conseguido afianzarse, al menos por el momento en vísperas de un nuevo evento electoral a nivel local.

(7) Véase su Instituciones políticas y constitucionales. Caracas, 1985, t. 1, pp. 597 y ss. 


\section{PRINCIPALES INNOVACIONES DE LA LEY ORGANICA DE REGIMEN MUNICIPAL DE 1988}

Se ha hecho notar el carácter de "adaptacionista» y racionalizador de esta norma. Juicio positivo que también es preciso encuadrar en la difícil realidad circundante, poco propicia a aventuras reformadoras. Resta resaltar aquellos aspectos de la ley que suponen un mayor margen de novedad, tanto desde el propio decurso de su ámbito territorial, como desde una visión aquende los mares.

\section{Las fuentes del ordenamiento local}

La Constitución había citado a los tres protagonistas esenciales en el marco normativo referido a las distintas corporaciones locales: la Constitución y sus principios organizativos; las leyes orgánicas nacionales de desarrollo y las leyes de los Estados.

Basamento al que se ha atenido de forma estricta la Ley Orgánica analizada, que en su artículo 14 precisa que los municipios y demás entidades locales se regirán por el siguiente orden de fuentes:

1. ${ }^{\circ}$ Por la Constitución de la República.

2. $\quad$ Por la presente Ley Orgánica.

$3 .^{\circ}$ Por las leyes orgánicas y ordinarias que les sean aplicables conforme a la Constitución (por ejemplo, la Ley Orgánica del Sufragio).

4. ${ }^{\circ}$ Por las leyes que dicten las asambleas legislativas (de los Estados) en desarrollo y ejecución de la Constitución y de la presente Ley Orgánica.

5. Por lo establecido en las Ordenanzas y demás instrumentos jurídicos municipales.

\section{Estructuras locales}

La Ley Orgánica va a reconocer (art. 13) cinco entidades territoriales locales: los municipios, los distritos municipales y metropolitanos, las parroquias y las mancomunidades. Se rompe de tal modo con el sistema precedente, caracterizado por su uniformidad (8).

Comenzando por los municipios, se atribuye su creación a la determinación de la asamblea legislativa del Estado respectivo,

(8) F. MODERNE: El problema..., p. 25 
dentro de unos requisitos mínimos que la ley estudiada ha suavizado respecto de lo vigente con anterioridad. La fuerte concentración poblacional venezolana ha supuesto que deban concurrir para tal evento, las siguientes circunstancias: una población no menor de 10.000 habitantes, un territorio determinado; un centro de población no menor de 2.500 habitantes y capacidad de generar recuisos propios, suficientes para atender los gastos de gobierno, administración y prestación de los servicios mínimos obligatorios. La "flexibilidad» añadida viene conformada por la posibilidad alternativa al mínimo de habitantes (10.000), si se da "la existencia de un grupo social asentado establemente con vínculos de vecindad permanenten; con lo que se ha abierto la facultad de establecer con criterios sociológicos las entidades locales, como unidades políticas primarias (9).

En cuanto al proceso de creación municipal, o de fusión de dos o más de los existentes, corresponde en cuanto a la iniciativa, a un 20 por 100 como mínimo de los ciudadanos inscritos en el censo electoral permanente, a la asamblea legislativa o a los Concejos municipales interesados, lo que no ofrece novedad sobre la legislación anterior; la decisión corresponde a la asamblea legislativa correspondiente, adoptada por el voto favorable de las dos terceras partes de sus integrantes. La Ley Orgánica atiende por primera vez al régimen transitorio derivado de la creación o fusión municipal, mediante la formación de una comisión para el deslinde de los asuntos patrimoniales y una junta administradora para llevar a buen puerto a la entidad emergente, en lo que quedaba de período municipal.

Se regula con mayor intensidad que en normativa precedente, las entidades locales supramunicipales. Comenzando por los distritos municipales como entidades locales de carácter público y con personalidad jurídica, formadas por la agrupación de dos o más municipios; si se trata de municipios rurales, se denominan distritos municipales y se llamarán distritos metropolitanos cuando la agrupación de municipios se realice "debido a la conurbación de sus centros poblados capitales y se hallen desarrollados en forma tal que lleguen a constituir una unidad urbana, social y económica con más de 250.000 habitantes».

Se eleva el mínimo anterior ( 100.000 habitantes) para estos distritos, que eran la pieza básica del escalón local en el ordenamiento predemocrático. Su creación se debía hacer mediante ley de la asamblea legislativa, previo nombramiento de una Comisión representativa. La creación de los mencionados distritos no extinguía a los municipios preexistentes, lo que implicaba una difícil

(9) BREWER-CARIAS: Introducción general..., p. 30 
relación competencial, puesto que existían competencias en dos niveles: el distrital municipal, para el que se perfilaba una lista de competencias de asunción obligatoria, y el nivel estrictamente municipal.

En cuanto a las mancomunidades, no existen grandes modificaciones sobre la Ley de 1978; dichas mancomunidades son entidades dotadas de personalidad jurídica, formadas mediante acuerdo entre dos o más municipios para la realización de determinados servicios municipales, con carácter voluntario de las correspondientes entidades municipales. No obstante, se prevé una forma peculiar de mancomunidad forzosa, en los supuestos de un servicio público municipal que tenga o requiera instalaciones ubicadas en jurisdicción de más de un municipio contiguo territorialmente, por un mismo organismo, entidad o empresa pública o privada.

Como entidades locales inframunicipales, en sustitución de los «municipios foráneos» aparecen las parroquias, como demarcaciones de carácter local, en el interior del territorio de un municipio, creadas con el objeto de descentralizar la Administración municipal, promover la participación ciudadana y la mayor prestación de los servicio públicos locales, pudiendo ser urbanas (coincidiendo con barrios, urbanizaciones, o sectores de ciudades, o incluso con ciudadanos interesados en número no inferior al 20 por 100 de los electores), o rurales (poblaciones, caseríos y aldeas separadas de la capital del municipio). Sus competencias las ostentarán mediante delegación del alcalde, acompañadas de los medios necesarios para su eficaz ejecución.

\section{El modelo organizativo y de funcionamiento}

El sistema de la Ley de 1978 se componía de una asamblea deliberante, denominada concejo municipal, y de un órgano ejecutivo llamado "administrador municipal» o "metropolitano», elegido por la asamblea local, siendo figura diferente del presidente del concejo. Como ha hecho notar MODERNE (10), el administrador era en definitiva un funcionario del municipio, designado previo concurso; el hecho de confiar la ejecución de las deliberaciones del concejo municipal a un funcionario y no al presidente electo del concejo, no podría sino debilitar a este último y convertirse en un semillero de conflictos. Luego en la práctica, todo el poder devenía a favor del órgano colegiado; es por ello que BREWER-CARIAS atribuye la mayor importancia a la reforma de la Ley de 1988, en cuanto a la superación del carácter colegiado del gobierno

(10) El problema..., p. 29. 
local (11), que había mostrado sus deficiencias, sobre todo por la difuminación de la responsabilidad gubernativa local.

La novedad radica, por lo tanto, en la emergencia del alcalde, como órgano ejecutivo del gobierno municipal; alcalde elegido por mayoría relativa, en votación universal, directa y secreta, con sujeción a lo dispuesto en la Ley Orgánica del Sufragio. Los requisitos para poder ser electo son los siguientes: ser venezolano por nacimiento o por naturalización, con no menos de tres años de residencia en el municipio o distrito; gozar de sus derechos civiles y políticos; estar inscrito en el registro electoral permanente y haber cumplido con el deber de votar (12). Cargo que será remunerado, con arreglo a las remuneraciones que se le fijen en la ordenanza del presupuesto.

En cuanto a sus atribuciones responden a su carácter de máximo órgano ejecutivo (dirigir el gobierno y administración municipal y ejercer la representación del municipio, ejercer la presidencia del concejo municipal de aquellos municipios de menos de $\mathbf{5 0 . 0 0 0}$ habitantes, sin que paradójicamente, tenga derecho a voto; ejecutar, dirigir e inspeccionar los servicios y obras municipales; dictar reglamentos de carácter interno; suscribir los contratos que celebre la entidad; ejercer la máxima autoridad en materia de administración de personal, etc.). Posee la obligación de cumplir y hacer cumplir las leyes, reglamentos y resoluciones emanadas de las autoridades nacionales y estatales.

El gobierno municipal se completa con el concejo municipal, que ejerce la rama deliberante, correspondiéndole «legislar sobre las materias de la competencia del municipio, y ejercer el control de la rama ejecutiva del gobierno municipal». La elección de los concejales se debía hacer por votación universal, directa y secreta, por el sistema de listas cerradas y bloqueadas en toda la circunscripción del municipio (13). El cargo tiene carácter honorífico, pudiendo percibir dietas por asistencia a sesiones del concejo.

Sus competencias oscilan entre la elección de su presidente en municipios de más de 50.000 habitantes, así como los nombramientos de los puestos de secretario, síndico y contralor del municipio; el sancionar las ordenanzas y aprobar el plan y programas de trabajo, el presupuesto de ingresos y gastos, etc.

En la Ley de 1978, el concejo era al mismo tiempo un órgano "legislativo» y un órgano "administrativo»; incluso una sentencia de

(11) Introducción general..., p. 65

(12) Como denota BREWER-CARIAS (Introducción general..., p. 67), «no precisa la ley cuándo es que debió haberse cumplido con este deber. ¿En el proceso electoral anterior o en todos?").

(13) Para BREWER-CARIAS: (Introducción general..., p. 26), el sistema de listas bloqueadas había provocado una crisis de representatividad a nivel local. 
la Corte Suprema de 4 de abril de 1978 declaraba que «los concejos municipales se reparten con el Congreso y las Asambleas Legislativas el poder de legislar, pero en un marco territorial más reducido y únicamente en lo que concierne a asuntos locales», produciendo una evidente confusión entre los «poderes» legislativo y administrativo (14). Este singular carácter de la ley de las ordenanzas municipales en relación con las materias propias de la competencia municipal (15), auténtica reserva reglamentaria a normas locales tal como se concibe en la dogmática europea, se reduce con el mandato constitucional a aquellas materias (sobra indicar que representan la gran mayoría) en las cuales hay una concurrencia de competencias entre los diversos niveles, respecto de las cuales corresponde a la ley nacional establecer el ámbito que corresponde a cada nivel. Se insistirá en esta cuestión posteriormente.

En cuanto al funcionamiento de los concejos no presenta ninguna originalidad, ateniéndose a lo que disponga el reglamento interno de la Cámara y asegurando el ejercicio de la democracia y del régimen parlamentario: publicidad, necesidad de levantar un acta en cuanto «instrumento público», deliberación con la presencia de una mayoría de los miembros, requiriéndose el voto de la mayoría absoluta de los concejales presentes para sancionar decisiones.

Existen otros cargos importantes en la organización local, con funciones que dada la pluralidad de órganos, de algún modo pueden solaparse entre sí. Debe citarse en primer lugar a la secretaría municipal, cargo designado por el concejo que debe permanecer durante el período de mandato del órgano que lo nombra; entre sus deberes se encuentra el elaborar las actas de las sesiones del concejo; refrendar las ordenanzas y demás instrumentos jurídicos que dicte; hacer llegar a los concejales las convocatorias para las sesiones extraordinarias; llevar con regularidad los libros, expedientes y documentos del concejo; expedir certificaciones de las actas; dirigir el personal y los trabajos de secretarias, etc.

Cargo similar por lo visto al secretario tal como se perfila por estos pagos, aunque contradictoriamente al venezolano se le exija sólo certificado de educación básica. La confusión puede producirse al existir otro cargo obligatorio, el síndico procurador municipal, que en los municipios de más de $\mathbf{5 0 . 0 0 0 ~ h a b i t a n t e s ~ d e b e ~ s e r ~}$ abogado y es designado por el concejo. Corresponde al síndico, el representar y defender, judicial y extrajudicialmente, los intereses

(14) Véase F. MODERNE: El problema..., p. 28.

(15) Al respecto BREWER-CARIAS: Jurisprudencia de las Corte Suprema 1939-1974 y Estudios de Derecho Administrativo, t. 11, Caracas, 1976, pp. 278 y ss. 
del municipio, en relación con los bienes y derechos municipales, así como en todo lo relacionado con el ordenamiento jurídico, sometiéndose a las instrucciones del alcalde y del concejo municipal (16); representar y defender al municipio en lo referente a derechos relacionados con ingresos públicos municipales, ostentando el carácter de inspector fiscal de la hacienda pública municipal; asesorar jurídicamente, cuando sea abogado, al alcalde y al concejo...

Por último, la ley orgánica determina que en cada municipio debía existir un cronista, cuyas funciones y requisitos de idoneidad para el ejercicio de su cargo, se debían de establecer en las ordenanzas.

\section{El plano de las competencias}

Las competencias locales se inscriben en un amplio listado abierto, y ello desde la propia Constitución, que en su artículo 30 enumera esas competencias, con carácter enunciativo. La Constitución establece como competencia de los municipios las materias propias de la vida local, adicionando que dichos municipios tienen la libre gestión de los intereses peculiares de la entidad, citándose a continuación las materias tales como urbanismo, abastos, circulación, cultura, salubridad, asistencia social, institutos populares de crédito, turismo y policía municipal.

La doctrina ha constatado la banalidad de estos listados, que en apariencia revelan un extenso ámbito competencial, pero que en la práctica se reducen en extrema medida; la concurrencia no menos permanente con competencias del poder nacional, ha hecho necesario una ley nacional que delimite ambos espacios conflictivos, obligando a la Corte Suprema de Justicia a una intervención, que con frecuencia ha supuesto la anulación de leyes nacionales por invadir competencias municipales. La consecuencia ha sido, tal como lo enuncia BREWER-CARIAS (17), que el ejercicio de competencias municipales se encuentra triplemente limitado: frente a los poderes residuales de los Estados, por los poderes implícitos del poder nacional y por los poderes concurrentes; a estas limitaciones debe agregarse la tendencia centralista del poder nacional.

La ley orgánica obliga que en todo municipio de más de 1.000 habitantes, los mismos, por sí mismos o mancomunados, deben garantizar a los vecinos los servicios minimos siguientes: alum-

(16) Para BREWER-CARIAS (Introducción general..., p. 85), "esta norma, sin duda inconveniente, dejó abierta una fuente de conflictos entre el alcalde y el concejo. En realidad, el síndico, por sus funciones, debe actuar conforme a las instrucciones del alcalde, y no del concejo...».

(17) Introducción general..., p. 43 
brado público y domiciliario, cementerio, recogida de residuos, limpieza diaria, abastecimiento domiciliario de agua potable, cloacas y alcantarillado, matadero, plaza pública, mercado público y atención primaria de la salud. En los municipios con población superior a 10.000 habitantes, además: parque público, biblioteca, plan de desarrollo urbano local y nomenclatura y señalización urbanas. En los municipios con población superior a 50.000 habitantes: protección civil, asistencia a la infancia y ancianos, planificación familiar, prevención y extinción de incendios, instalaciones deportivas de uso público y matadero. En los municipios con población superior a 100.000 habitantes: servicio de transporte público urbano de pasajeros, protección del medio ambiente, control de alimentos y bebidas y tratamiento de residuos.

En cuanto a la prestación de los servicios públicos municipales pueden ser gestionados de la siguiente forma: $1 .^{\circ} \mathrm{El}$ municipio en forma directa; $2 .^{\circ}$ institutos autónomos municipales, «mediante delegación»; $3 .^{\circ}$ empresas, fundaciones, asociaciones civiles y otros organismos descentralizados del municipio, mediante contrato; $4{ }^{\circ}$ organismos de cualquier naturaleza de carácter nacional o estatal, mediante contrato; $5 .^{\circ}$ concesión otorgada mediante licitación pública.

La ley orgánica establece asimismo para los municipios, la posibilidad de celebrar acuerdos o convenios para fines de interés común. Incluso se prevé que los municipios puedan constituir una asociación civil sin fines de lucro y con personalidad jurídica propia, de carácter nacional, con objeto de promover y facilitar el desarrollo integral de los municipios.

\section{La hacienda pública municipal}

MODERNE había hecho notar (18) que entre los grandes obstáculos que podían comprometer el éxito de la experiencia de la Ley de 1979, se encontraba, en primer lugar, los problemas financieros municipales, problemas que no han hecho más que agravarse con el paso del tiempo, en estrecha conexión con el deterioro económico del país en su totalidad.

En cuanto a los impuestos municipales, los determinados en la Constitución y que representan su fuente principal de ingresos son, en primer lugar, el impuesto de "patente de industria y comercio», que impone las actividades lucrativas que se realizan en el territorio de cada municipio, teniendo como base imponible el total de las ventas $y$ operaciones efectuadas por el contribuyente el año anterior.

(18) El problema..., p. 33 
Se halla a continuación el impuesto sobre inmuebles urbanos, que además se configura en el único impuesto territorial existente, aunque la situación catastral sea harto deficiente.

En tercer lugar, el denominado patente de vehículos, que grava el uso de los mismos, hallándose la base imponible determinada por la clasificación de los diversos vehículos.

Se cita a continuación el impuesto sobre espectáculos públicos, que recae sobre los billetes de tales espectáculos; el impuesto sobre apuestas lícitas, con motivo de espectáculos públicos permitidos, no debiendo exceder del 5 por 100 del total de lo aportado, cuando se origine en sistemas de juego establecidos nacionalmente por algún instituto oficial.

Debe hacerse notar que la ley orgánica autoriza a los municipios a contratar la recaudación de estos tributos con el ejecutivo nacional o estatal, empresas públicas o privadas de reconocida solvencia, «siempre y cuando ello asegure una recaudación más eficaz y a menos costo".

Si no existen novedades reseñables en cuanto a las tasas y a las contribuciones especiales, si que merece citarse la fuente de ingresos derivada del "situado municipal», que viene a representar la participación de los Estados en los ingresos municipales, tratando de este modo de remediar la extrema debilidad de los recursos municipales. De acuerdo con la ley orgánica de coordinación del "situado constitucional» con los planes administrativos desarrollados por el poder nacional, en las leyes de presupuestos de los Estados, se debía incorporar una partida, en tanto «situado municipal», no menor del 12,5 por 100 ni mayor del 20 por 100 del situado constitucional no sujeto a coordinación para ser distribuida entre los concejos municipales.

El situado municipal debía distribuirse entre los municipios en un 50 por 100 por partes iguales, y el 50 por 100 restante en proporción a la población de cada entidad, debiéndose invertir en la construcción de obras y adquisición de equipos para la prestación de servicios públicos y para los gastos imprescindibles para el funcionamiento de dichos servicios.

En cuanto a la elaboración $y$ aprobación del presupuesto, corresponde al alcalde presentar al concejo el proyecto de ordenanza del presupuesto correspondiente al ejercicio fiscal siguiente; dicho proyecto se somete a la consideración del concejo, por lo menos con cuarenta y cinco días de anticipación al inicio del ejercicio. El concejo puede alterar las partidas presupuestarias, pero no autorizará gastos que excedan del total de las estimaciones de ingresos del respectivo proyecto de ordenanza de presupuesto.

En cuanto al sistema de control, dicho control fiscal y adminis- 
trativo de los municipios corresponde, de acuerdo con la Constitución y la ley orgánica descrita, tanto a la Contraloría General de la República (19) como a las contralorías municipales, donde existan.

Las contralorías municipales, bajo la dirección del contralor nombrado por el concejo mediante concurso, ejerce el control, la vigilancia y la fiscalización de los ingresos, gastos y bienes públicos municipales; deben existir obligatoriamente en los municipios y distritos municipales o metropolitanos con una población superior a 100.000 habitantes, estando obligado el contralor a remitir anualmente a la Contraloría General de la República al finalizar el período fiscal, un informe de sus actuaciones y de las gestiones administrativas del municipio, una relación de ingresos y gastos de éste, los estados de ejecución del presupuesto, los balances contables $y$ el inventario anual actualizado de los bienes del respectivo municipio.

\section{La participación política en el régimen municipal}

Siguiendo a BREWER-CARIAS (20) los mecanismos de participación de la comunidad en la conducción de los asuntos locales, pueden agruparse del siguiente modo: los de participación política; los de participación en asuntos vecinales, y los de participación en defensa de los bienes municipales.

En cuanto al primer punto, para el ejercicio del derecho electoral se requiere la vecindad, en el sentido de acreditarse la residencia (21), mediante el acto administrativo de incorporación en el registro electoral permanente.

En la ley orgánica se ha aceptado la posibilidad de un referéndum municipal, convocado por el concejo, con el fin de consulta de las ordenanzas, cuando así lo decida la cámara, o lo solicite un 10 por 100 de los vecinos inscritos en el registro electoral permanente. El concejo supremo electoral ejerce la organización y vigilancia de estos procedimientos de referéndum.

Existe asimismo un interesante mecanismo que permite a los vecinos la iniciativa legislativa en materia de ordenanzas, así como la posibilidad de solicitar a los concejos municipales la reconsideración de ciertas ordenanzas.

En cuanto a la participación en asuntos vecinales, puede aludirse a la posibilidad de concejos o cabildos abiertos; la ley orgánica establece al respecto que cada tres meses por lo menos, los

(19) En el Diccionario de la Lengua Española de la Real Academia Española (Madrid, 1989). se señala lo siguiente sobre el término "Contraloría»: "En algunos países de América, servicio encargado de examinar la legalidad y corrección de los gastos públicos.»

(20) Introducción general..., pp. 134 y ss.

(21) Residentes pueden ser tanto los nacionales como los extranjeros con más de diez años de residencia en el país y con más de un año de residencia en el municipio. 
concejos y cabildos deben celebrar una sesión en la cual se deben considerar las materias que los vecinos hayan solicitado por escrito y con quince días de anticipación por lo menos, a la fecha de la reunión. En la sesión correspondiente, el público asistente puede formular preguntas y emitir opiniones y hace proposiciones sobre las materias inscritas en el orden del día.

Se perfilan unas comisiones de vecinos encargadas de vigilar el buen funcionamiento en su territorio, de determinados servicios públicos: educativos, asistenciales, de policía, de cloacas, de aseo urbano, de abastecimiento, mercados y control de precios, de cementerios y servicios funerarios, de transportes públicos, etc.

La figura central con todo, lo constituyen las asociaciones de vecinos que tratan de responder a las insuficiencias demostrables de los concejos municipales. Reconocidas en la precedente Ley de 1978, las asociaciones vecinales son de carácter privado, actuando como grupo de presión, de forma especial en las grandes conurbanizaciones, de forma singular en los barrios periféricos de Caracas.

La presente ley orgánica ha continuado con el espíritu promocional de estas asociaciones, imponiendo a los municipios el deber de favorece su desarrollo, facilitándoles la más amplia información sobre sus actividades, así como el uso de los medios públicos y el acceso a las ayudas económicas para la realización de sus fines.

Sociológicamente, su ámbito territorial es la urbanización o el barrio, aunque también existe a nivel del global municipio rural. La ley orgánica ha sido particularmente flexible en cuanto a los requisitos para su constitución, aunque se remita al Reglamento para establecer «el número mínimo de sus integrantes». En cuanto a su ámbito territorial, la ley orgánica señala que éste debe ser determinado por la oficina municipal que tenga a su cargo la planificación urbana.

En cuanto a las funciones de las asociaciones de vecinos giran en torno a un eje: La defensa de los intereses colectivos, lo que implica una auténtica multiplicidad de fines, realizados y perseguidos como organismos autónomos no dependientes de las autoridades muncipales. En realidad, se trata de personas jurídicas de derecho privado, cuya personalidad jurídica la adquieren como sociedades civiles sin fines de lucro, mediante la inscripción de su acta constitutiva y estatutos en la correspondiente oficina subalterna de registro; siendo igualmente asociaciones de carácter voluntario, no ejerciendo autoridad y cuyos directivos son elegidos por la propia comunidad vecinal, sin que exista intervención alguna de la administración municipal. 
En todo caso (22), la permanencia distingue a las asociaciones de vecinos de los comités de lucha de muchos barrios marginales, de existencia circunstancial.

La ley orgánica, finalmente, establece dos supuestos de participación de los vecinos en defensa de los bienes municipales. En primer lugar atribuye la legitimación a cualquier vecino del municipio, para solicitar la intervención del ministerio público para que inste al alcalde a que tome las medidas pertinentes para el reconocimiento de su propiedad o posesión de ejidos e inmuebles municipales enajenados con violación de lo dispuesto en la Constitución, ordenanzas y leyes, o sean detentadas sin causa o justo título.

En segundo lugar, se atribuye la legitimación a cualquier vecino del municipio, para solicitar del concejo municipal la declaración de inexistencia de una donación o de un usufructo de bienes muebles del dominio privado del municipio, realizada en contravención de la ley orgánica.

\section{EL DIFICIL DECURSO DE LA LEY}

MODERNE, en los primeros momentos aplicativos de la Ley de 1978, emitía un juicio particularmente excéptico (23): "Las estructuras administrativas no están todavía adaptadas a las múltiples tareas que reclaman los municipios. La formación del personal, la modernización de los servicios públicos esenciales, una planificación local racional, la mejora del sistema fiscal, una atención mayor a los problemas del urbanismo, constituyen un vasto programa que exigirá muchos esfuerzos y paciencia...»

Se ha mentado la clara voluntad de la ley orgánica de régimen municipal venezolana de 1988, por insertarse en unos parámetros de racionalidad, tratando de superar las disfuncionalidades derivadas de una ley cuyo mayor defecto consistió precisamente en su inaplicación. Pretendiendo de igual manera recoger las experiencias proporcionadas por sistemas cercanos, sin romper con ello con determinadas señas de identidad propias.

El interrogante se plantea si estas excelentes intenciones podrán germinar en un terreno tan árido y repleto de problemas, como en la actualidad significa Venezuela. Se ha hecho reiterada mención a la crisis financiera, que tan directamente atañe al mismo erario municipal, y podía también aludirse a la crisis del propio sistema democrático, incapaz por el momento de encontrar soluciones que afronten la problemática estructural. Resta con todo emitir un juicio netamente positivo, merecido por el esfurzo denotable en la ley de régimen municipal examinada.

(22) BREWER-CARIAS: Introducción general..., p. 145

(23) El problema..., p. 34. 
